\title{
Synchronous Parallel Database Transformations
}

\author{
Klaus-Dieter Schewe ${ }^{1}$ and Qing Wang $^{2}$ \\ 1 Software Competence Center Hagenberg and Johannes-Kepler-University Linz, \\ Austria \\ kd.schewe@scch.at, kd.schewe@faw.at, kdschewe@acm.org \\ 2 Department of Information Science, University of Otago, New Zealand \\ qing. wang@otago.ac.nz
}

\begin{abstract}
The DB-ASM thesis states that every database transformation can be expressed by a variant of Abstract State Machines. These machines permit unbounded parallelism only on the finite database part of a state. This paper generalises this work by permitting unbounded parallelism on the algorithmic part of the state as well. The "parallel DB-ASM"-thesis results from combining Gurevich's parallel ASM thesis with the DB-ASM thesis. In doing so, it turns out that the postulates for synchronous parallel database transformations can be significantly simplified compared with the seminal work of Gurevich. The key idea is to generalise the notion of bounded exploration witnesses allowing them to include special non-ground terms.
\end{abstract}

Keywords: Abstract State Machine, database transformation, bounded exploration, synchronous parallelism.

\section{Introduction}

Abstract State Machines (ASMs) has established themselves as an elegant and powerful instrument in the development of software systems 3 . However, their origin goes back to the fundamental question to give language-independent definition of the notion of (sequential) algorithm. So, the "ASM thesis" is considered to be an analogue to Church's thesis, which clarified the notion of computable function.

The ASM thesis actually refers to more than one result. The latest form of the sequential ASM thesis refers to Gurevich's seminal work on sequential algorithms, which he defined by three simple, intuitive postulates [6], whereas the parallel ASM thesis refers to the generalisation by Blass and Gurevich to (synchronous) parallel algorithms [2].

In our own previous work we adapted the sequential ASM thesis to characterise database transformations in general by a variant of ASMs called DB-ASMs [7. The core of the approach is similar to Gurevich's seminal work: provide a language-independent definition through a set of intuitive postulates, define formally an abstract machine model, and prove that the postulates are exactly captured by the machine model. On these grounds it was then possible to tailor 
the model to specific data model, e.g. XML [8], and to pick up partial updates [9] thereby extending previous work on applicative algebras in [5].

This paper is dedicated to the problem to remove the restriction that only sequential algorithms are exploited in the DB-ASM thesis. That is, we want to generalise our previous work to the case of synchronous parallel database transformations in general. The corresponding parallel ASM thesis [2] is significantly more complicated than the sequential sequential ASM thesis, as it involves several postulates to handle the synchronisation of different parallel branches of a computation.

We first review the DB-ASM thesis in Section 2, Then, Section 3 contains the core of the paper addressing the formal generalisation to parallel synchronous database ASMs. We conclude with a brief summary and outlook.

\section{The Database-ASM Thesis}

In our work on DB-ASMs [7] we adopted the approach of Gurevich that led to the sequential ASM thesis [6], i.e. we defined database transformations in a language-independent way by five intuitive postulates, which we shall briefly review in this section. We also present DB-ASMs, the variant of ASMs that captures exactly these database transformations.

\subsection{Database Transformations}

We will formally introduce the five postulates for database transformations: the sequential time postulate, the abstract state postulate, the background postulate, the bounded exploration postulate, and the bounded non-determinism postulate. An object satisfying these postulates will be called a database transformation.

As in [6] we assume that a database transformation same as any algorithm proceeds step-wise on a set of states. This leads to the following sequential state postulate, which (with some minor modifications) is the same as in the Gurevich's ASM thesis.

Postulate 1 (sequential time postulate). A database transformation $t$ is associated with a non-empty set of states $\mathcal{S}_{t}$ together with non-empty subsets $\mathcal{I}_{t}$ and $\mathcal{F}_{t}$ of initial and final states, respectively, and a one-step transition relation $\tau_{t}$ over $\mathcal{S}_{t}$, i.e. $\tau_{t} \subseteq \mathcal{S}_{t} \times \mathcal{S}_{t}$.

The second postulate concerns the states. Its first part is a simple adaptation of the abstract state postulate in [6] to the presence of final states and the fact that one-step transition is a binary relation. The second part is specific to databases. The rationale is that the states of a database transformation should capture databases that are defined over some database schema.

Postulate 2 (abstract state postulate). All states $S \in \mathcal{S}_{t}$ of a database transformation $t$ are structures over the same signature $\Sigma_{t}$, and whenever $\left(S, S^{\prime}\right)$ $\in \tau_{t}$ holds, the states $S$ and $S^{\prime}$ have the same base set $B$. The sets $\mathcal{S}_{t}, \mathcal{I}_{t}$ and $\mathcal{F}_{t}$ 
are closed under isomorphisms, and for $\left(S_{1}, S_{1}^{\prime}\right) \in \tau_{t}$ each isomorphism $\sigma$ from $S_{1}$ to $S_{2}$ is also an isomorphism from $S_{1}^{\prime}$ to $S_{2}^{\prime}=\sigma\left(S_{1}^{\prime}\right)$ with $\left(S_{2}, S_{2}^{\prime}\right) \in \tau_{t}$.

Furthermore, the signature $\Sigma_{t}$ is composed as a disjoint union out of a signature $\Sigma_{d b}$ (called database part), an signature $\Sigma_{a}$ (called algorithmic part), and a finite set of bridge function symbols each with a fixed arity, i.e. $\Sigma_{t}=$ $\Sigma_{d b} \cup \Sigma_{a} \cup\left\{f_{1}, \ldots, f_{\ell}\right\}$. The base set of a state $S$ is $B=B_{d b}^{e x t} \cup B_{a}$ with interpretation of function symbols in $\Sigma_{d b}$ and $\Sigma_{a}$ over $B_{d b} \subseteq B_{d b}^{e x t}$ and $B_{a}$, respectively, with $B_{d b}$ depending on the state $S$. The interpretation of a bridge function symbol of arity $k$ defines a function from $B_{d b}^{k}$ to $B_{a}$. With respect to such states $S$ the restriction to $\Sigma_{d b}$ is a finite structure, i.e. $B_{d b}$ is finite.

As states are structures, terms $t$ with variables $f r(t)=\left\{x_{1}, \ldots, x_{n}\right\}$ can be evaluated in them for a given variable assignment $\zeta=\left\{x_{1} \mapsto a_{1}, \ldots, x_{n} \mapsto a_{n}\right\}$ with elements $a_{1}, \ldots, a_{n}$ of the base set of the structure. We use the notation $v a l_{S, \zeta}(t)$ for the value in the base set of the state $S$ that results from evaluating term $t$ in $S$ with respect to $\zeta$.

The third postulate refers to the background of a computation, which contains everything that is needed to perform the computation, but is not yet captured by the state. For instance, truth values and their connectives, and a value $\perp$ to denote undefinedness constitute necessary elements in a background. The background is the same as in [2].

Postulate 3 (background postulate). Each state of a database transformation $t$ must contain

- an infinite set of reserve values,

- truth values and their connectives, the equality predicate, the undefinedness value $\perp$, and

- a background class $\mathcal{K}$ defined by a background signature $V_{K}$ that contains at least a binary tuple constructor $(\cdot)$, a multiset constructor $\langle\cdot\rangle$, and function symbols for the following operations: pairing and projection for pairs, empty multiset \langle\rangle , singleton $\langle x\rangle$, binary multiset union $\uplus$, general multiset union $\biguplus x, A s S e t$, and $\mathbf{I} x$ ("the unique") on multisets.

The bounded exploration postulate in the sequential ASM thesis in 6] uses a finite set of ground terms as bounded exploration witness in the sense that whenever states $S_{1}$ and $S_{2}$ coincide over this set of ground terms the update set produced by the sequential algorithm is the same in these states. The intuition behind the postulate is that only the part of a state that is given by means of the witness will actually be explored by the algorithm.

The fact that only finitely many locations can be explored remains the same for database transformations. However, permitting parallel accessibility within the database part of a state forces us to slightly change our view on the bounded exploration witness. For this we need access terms.

Definition 1. An access term is either a ground term $\alpha$ or a triple $(f, \beta, \alpha)$ of terms, the variables $x_{1}, \ldots, x_{n}$ in which refer to the arguments of $f \in \Sigma_{d b}$. The interpretation of $(f, \beta, \alpha)$ in a state $S$ is the set of locations 


$$
\left\{f\left(a_{1}, \ldots, a_{n}\right) \mid \operatorname{val}_{S, \zeta}(\beta)=\operatorname{val}_{S, \zeta}(\alpha) \text { with } \zeta=\left\{x_{1} \mapsto a_{1}, \ldots, x_{n} \mapsto a_{n}\right\}\right\} .
$$

Structures $S_{1}$ and $S_{2}$ coincide over a set $T$ of access terms iff the interpretation of each $\alpha \in T$ and each $(f, \beta, \alpha) \in T$ over $S_{1}$ and $S_{2}$ are equal.

For a database transformation $t$, a function symbol $f$ of arity $n$ in the state signature and values $a_{1}, \ldots, a_{n}, v$ in the base set of states $f\left(a_{1}, \ldots, a_{n}\right)$ is called a location. An update is a pair $(\ell, v)$, where $\ell$ is a location. $\Delta(t, S)$ denotes the set of updates that $t$ will perform in state $S$ (this is well defined [7]).

Due to our request that the database part of a state is always finite there will be a maximum number $m$ of elements that are accessible in parallel. Furthermore, there is always a number $n$ such that $n$ variables are sufficient to describe the updates of a database transformation, and $n$ can be taken to be minimal. Then for each state $S$ the upper boundary of exploration is $\mathcal{O}\left(m^{n}\right)$, where $m$ depends on $S$. Taking these together we obtain our fourth postulate.

Postulate 4 (bounded exploration postulate). For a database transformation $t$ there exists a fixed, finite set $T$ of access terms of $t$ (called bounded exploration witness $)$ such that $\Delta\left(t, S_{1}\right)=\Delta\left(t, S_{2}\right)$ holds whenever the states $S_{1}$ and $S_{2}$ coincide over $T$.

The last postulate bounds non-determinism in a database transformation to choices depending on the database part.

Postulate 5 (bounded non-determinism postulate). For a database transformation $t$, if there are states $S_{1}, S_{2}$ and $S_{3} \in \mathcal{S}_{t}$ with $\left(S_{1}, S_{2}\right) \in \tau_{t},\left(S_{1}, S_{3}\right) \in \tau_{t}$ and $S_{2} \neq S_{3}$, then there exists a non-ground access term of the form $(\beta, \alpha)$ in the bounded exploration witness of $t$.

In particular, if we ignore the database part by assuming $\Sigma_{d} b=\emptyset$, then a database transformations degenerates to a sequential algorithm in the sense of Gurevich [6. There would be no non-determinism according to Postulate 5] and bounded exploration witnesses would only contain ground terms.

\subsection{Database Abstract State Machines}

As for ASMs, DB-ASMs are defined by rules which we sketch here (for formal details see [7]):

- If $t_{0}, \ldots, t_{n}$ are terms over $\Sigma$, and $f$ is a $n$-ary function symbol in $\Sigma$, then $f\left(t_{1}, \ldots, t_{n}\right):=t_{0}$ is a rule $r$ in $\mathcal{R}$ called assignment rule.

- If $\varphi$ is a Boolean term and $r^{\prime} \in \mathcal{R}$ is a DB-ASM rule, then if $\varphi$ then $r^{\prime}$ endif is a rule $r$ in $\mathcal{R}$ called conditional rule.

- If $\varphi$ is a Boolean term with only database variables, $\left\{x_{1}, \ldots, x_{k}\right\} \subseteq \operatorname{fr}(\varphi)$ and $r^{\prime} \in \mathcal{R}$ is a DB-ASM rule, then forall $x_{1}, \ldots, x_{k}$ with $\varphi$ do $r^{\prime}$ enddo is a rule $r$ in $\mathcal{R}$ called forall rule.

- If $r_{1}, \ldots, r_{n}$ are rules in $\mathcal{R}$, then the rule $r$ defined as par $r_{1} \ldots r_{n}$ endpar is a rule in $\mathcal{R}$, called parallel rule. 
- If $\varphi$ is a Boolean term with only database variables, $\left\{x_{1}, \ldots, x_{k}\right\} \subseteq \operatorname{fr}(\varphi)$ and $r^{\prime} \in \mathcal{R}$ is a DB-ASM rule, then choose $x_{1}, \ldots, x_{k}$ with $\varphi$ do $r^{\prime}$ enddo is a rule $r$ in $\mathcal{R}$ called choice rule.

- If $r_{1}, r_{2}$ are rules in $\mathcal{R}$, then the rule $r$ defined as seq $r_{1} \quad r_{2}$ endseq is a rule in $\mathcal{R}$, called sequence rule.

- If $r^{\prime} \in \mathcal{R}$ is a DB-ASM rule and $\theta$ is a location function that assigns location operators $\rho$ to terms $t$ with $\operatorname{var}(t) \subseteq f r\left(r^{\prime}\right)$, then let $\theta(t)=\rho$ in $r^{\prime}$ endlet is a rule $r \in \mathcal{R}$ called let rule.

Here a location function $\rho$ on the set $M(D)$ of all non-empty multisets over a domain $D$ is defined by a unary function $f_{\alpha}: D \rightarrow D$, a commutative and associative binary operation $\odot$ over $D$, and a unary function $f_{\beta}: D \rightarrow D$, which define $\rho(m)=f_{\beta}\left(f_{\alpha}\left(b_{1}\right) \odot \cdots \odot f_{\alpha}\left(b_{n}\right)\right)$ for $m=\left\langle b_{1}, \ldots, b_{n}\right\rangle \in M(D)$.

The key results on database transformations are the following two theorems (for a proof see [7]):

Theorem 1. Each DB-ASM $\mathcal{M}$ defines a database transformation $t$ with the same signature and background as $\mathcal{M}$.

Theorem 2. For every database transformation $t$ there exists an equivalent $D B-A S M \mathcal{M}$ with the same background.

\section{Deterministic Synchronous Parallel Database Transformations}

In a nutshell the model of DB-ASMs combines computations on finite databases with any sequential algorithm working on the algorithmic part of the states. This generalises reflective relational machines (see [1]) in that computations refer to sequential ASMs rather than Turing machines. As shown in 8 for the case of XML the access to the database part can be enhanced by adding more complex logical constructs.

However, the algorithms incorporated into DB-ASMs are only sequential in the sense that there is no way to have arbitrarily many parallel branches in a computation, the number of which is not determined a priori by the algorithm, but depends on the state. Our aim is to remove this restriction.

\subsection{Modified Postulates}

One way to do this would be to redo the work that led to the postulates for database transformations starting from the postulates for parallel algorithms in [2] instead of those for sequential algorithms in 6]. However, we will proceed differently by first shedding more light into the extensions in our postulates compared with the sequential ASM thesis:

- The key difference in the sequential time postulate is the support for nondeterminism. If for the time being we concentrate on deterministic database transformations, we could have kept Gurevich's postulate without change. 
- As a consequence, there would be no need for the bounded non-determinism postulate.

- The use of meta-finite states in the abstract state postulates is not more than a restriction needed for databases, and the background postulates only makes the need for certain values and operations obvious.

- So, the key difference lies in the extension of the bounded exploration witnesses.

Checking the proofs for the DB-ASM thesis, i.e. for Theorems 1 and 2 , shows that the restriction of access terms to the database part of the structure is not really necessary. Therefore, we will define (deterministic, synchronous) parallel database transformations (parDB transformations for short) by four postulates: a slightly modified sequential time postulate 6 , the abstract state postulate 2, the background postulate 3, and a new bounded exploration postulate 4, in which the notion of access term is generalised.

Postulate 6 (sequential time postulate). A database transformation $t$ is associated with a non-empty set of states $\mathcal{S}_{t}$ together with a non-empty subset $\mathcal{I}_{t}$ of initial states, and a one-step transition function $\tau_{t}: \mathcal{S}_{t} \rightarrow \mathcal{S}_{t}$.

For the bounded exploration postulate we only have to generalise the notion of access term letting comprehensions not only refer to the database part of the signature.

Definition 2. An access term is either a ground term $\alpha$ or a triple $(f, \beta, \alpha)$ of terms, the variables $x_{1}, \ldots, x_{n}$ in which refer to the arguments of $f \in \Sigma$. The interpretation of $(f, \beta, \alpha)$ in a state $S$ is the set of locations

$$
\left\{f\left(a_{1}, \ldots, a_{n}\right) \mid \operatorname{val}_{S, \zeta}(\beta)=\operatorname{val}_{S, \zeta}(\alpha) \text { with } \zeta=\left\{x_{1} \mapsto a_{1}, \ldots, x_{n} \mapsto a_{n}\right\}\right\} .
$$

Structures $S_{1}$ and $S_{2}$ coincide over a set $T$ of access terms iff the interpretation of each $\alpha \in T$ and each $(\beta, \alpha) \in T$ over $S_{1}$ and $S_{2}$ are equal.

\subsection{Examples}

Let us now look at some examples for synchronous parallel database transformations. In the following we look at cases where there is obviously unbounded parallelism involved in the sense that the parallel branches are not determined by the algorithm, but by the state (for a detailed discussion see [2]). Thus, we emphasize that these algorithms are captured by the generalised bounded exploration postulate involving non-ground terms in the bounded exploration witnesses.

Example 1. Let us look at the example of graph inversion, which was also used in 2] to motivate the postulates for parallel algorithms. Here we can assume two database relations in the signature $\Sigma_{d b}$, the unary relation NoDE, and the binary relation EDGE (let us simplify the example by ignoring the bridge functions). The algorithm can be simply expressed by the rule 
forall $x, y$ with $\operatorname{NoDE}(x) \wedge \operatorname{NodE}(y)$

do

enddo

$\operatorname{EdgE}(x, y):=\neg \operatorname{EdGE}(x, y)$

Here the exploration boundary witness can be taken as

$$
T=\{(\operatorname{Edge}, \operatorname{Node}(x), \operatorname{Node}(y))\} .
$$

Example 2. Take the rather well known LCR algorithm for leader election in a ring. Again, let us keep the presentation simple by ignoring the bridge functions. Then we need a unary function UID, which maps node identifiers onto numerical values, a unary function SEND, which does the same, an incoming message function IN that maps node identifiers to numerical values or unknown, a unary function STATUS that maps node identifiers the values unknown or leader, a static unary function VAL mapping node identifiers to numerical values, and a constant $N$ for the number of nodes in the ring.

In an initial state we have $\operatorname{UID}(i)=\operatorname{VAL}(i), \operatorname{SEND}(i)=\operatorname{VAL}(i), \operatorname{IN}(i)=$ unknown, and $\operatorname{status}(i)=$ unknown for all $i$.

Then the database transformation algorithm can be specified as follows:

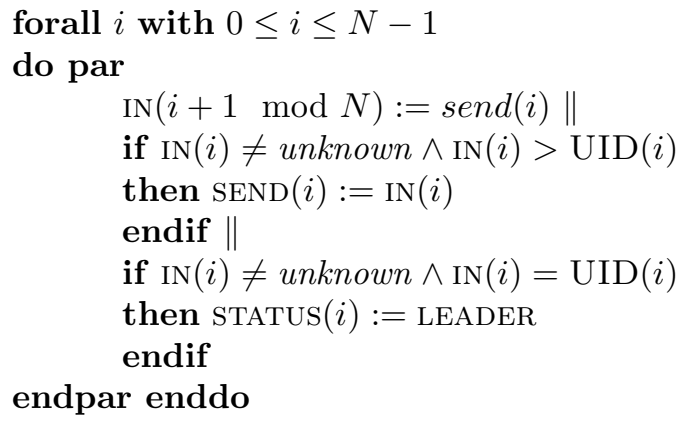

Here, the bounded exploration witness $T$ contains three access terms (UID, $\alpha(i)$, true), (IN, $\alpha(i)$, true) and (SEND, $\alpha(i)$, true), in which $\alpha(i)$ is defined as $i \geq 0 \wedge i<N$.

\subsection{Synchronous Parallel Database Abstract State Machines}

As for DB-ASMs we only define the rules for synchronous parallel database Abstract State Machines (parDB-ASMs) - the definition of the machines as such is the same as for DB-ASMs [7]:

- If $t_{0}, \ldots, t_{n}$ are terms over $\Sigma$, and $f$ is a $n$-ary function symbol in $\Sigma$, then $f\left(t_{1}, \ldots, t_{n}\right):=t_{0}$ is a rule $r$ in $\mathcal{R}$ called assignment rule.

- If $\varphi$ is a Boolean term and $r^{\prime} \in \mathcal{R}$ is a DB-ASM rule, then if $\varphi$ then $r^{\prime}$ endif is a rule $r$ in $\mathcal{R}$ called conditional rule. 
- If $\varphi$ is a Boolean term with $\left\{x_{1}, \ldots, x_{k}\right\} \subseteq f r(\varphi)$ and $r^{\prime} \in \mathcal{R}$ is a DB-ASM rule, then forall $x_{1}, \ldots, x_{k}$ with $\varphi$ do $r^{\prime}$ enddo is a rule $r$ in $\mathcal{R}$ called forall rule.

- If $r_{1}, \ldots, r_{n}$ are rules in $\mathcal{R}$, then the rule $r$ defined as par $r_{1} \ldots r_{n}$ endpar is a rule in $\mathcal{R}$, called parallel rule.

- If $r_{1}, r_{2}$ are rules in $\mathcal{R}$, then the rule $r$ defined as seq $r_{1} \quad r_{2}$ endseq is a rule in $\mathcal{R}$, called sequence rule.

- If $r^{\prime} \in \mathcal{R}$ is a DB-ASM rule and $\theta$ is a location function that assigns location operators $\rho$ to terms $t$ with $\operatorname{var}(t) \subseteq f r\left(r^{\prime}\right)$, then let $\theta(t)=\rho$ in $r^{\prime}$ endlet is a rule $r \in \mathcal{R}$ called let rule.

So, the only extension is the scope of variables in the forall rules, whereas choice rules have been discarded. First, we can generalise Theorem 1 .

Theorem 3. Each parDB-ASM $\mathcal{M}$ defines a synchronous parallel database transformation $t$ with the same signature and background as $\mathcal{M}$.

Proof (sketch). We have to show that the four postulates for synchronous parallel database transformations are satisfied. As for the sequential time and background postulates 6 and 3 , these are already built into the definition of a parDB-ASM. The same holds for the abstract state postulate 2 as far as the definition of states is concerned, and the preservation of isomorphisms is straightforward. Thus, we have to concentrate on the bounded exploration postulate 4

For this we note that assignment rules within a parDB-ASM rule $r$ are decisive for an update set $\Delta(r, S)$ of update sets over any state $S$. If $f\left(t_{1}, \ldots, t_{n}\right):=t_{0}$ is an assignment rule occurring within $r$, and $v^{\prime} l_{S, \zeta}\left(t_{i}\right)=v a l_{S^{\prime}, \zeta}\left(t_{i}\right)$ holds for all $i=0, \ldots, n$ and all variable assignments $\zeta$ that have to be considered, then we obtain $\Delta(r, S)=\Delta\left(r, S^{\prime}\right)$.

We use this to define a bounded exploration witness $T$. If $t_{i}$ is ground, we add the access term $\alpha=t_{i}$ to $T$. If $t_{i}$ is not ground, then the corresponding assignment rule must appear within the scope of a forall rule introducing the variables in $t_{i}$. Thus, variables in $t_{i}$ are bound by a Boolean term $\varphi$, i.e. for $f r\left(t_{i}\right)=\left\{x_{1}, \ldots, x_{k}\right\}$ the relevant variable assignments are $\zeta=\left\{x_{1} \mapsto b_{1}, \ldots, x_{k} \mapsto b_{k}\right\}$ with $\operatorname{val}_{S, \zeta}(\varphi)=$ true. Bringing $\varphi$ into a form that only uses conjunction, negation and existential quantification with atoms $\beta_{i}=\alpha_{i}$ $(i=1, \ldots, \ell)$, we can extract a set of access terms $\left\{\left(\beta_{1}, \alpha_{1}\right), \ldots,\left(\beta_{\ell}, \alpha_{\ell}\right)\right\}$ such that if $S$ and $S^{\prime}$ coincide on these access terms, they will also coincide on the formula $\varphi$. This is possible, as we evaluate access terms by sets, so conjunction corresponds to union, existential quantification to projection, and negation to building the (finite) complement. We add all the access terms $\left(\beta_{1}, \alpha_{1}\right), \ldots,\left(\beta_{\ell}, \alpha_{\ell}\right)$ to $T$.

\subsection{The Equivalence Result for Parallel Database ASMs}

Next we want to generalise Theorem 2 .

Theorem 4. For every synchronous parallel database transformation t there exists an equivalent parDB-ASM $\mathcal{M}$ with the same background. 
As for the DB-ASM thesis ths theorem follows from a sequence of lemmata, which we will sketch now. We start again providing the key link from updates as implied by the state transitions to parDB-ASM rules.

Definition 3. Let $T$ be a bounded exploration witness for the parallel database transformation $t$. A term that is constructed out of the subterms of $\alpha \in T$ and variables $x_{1}, \ldots, x_{k}$, for which there are access terms $\left(f_{1}, \beta_{1}, \alpha_{1}\right), \ldots,\left(f_{\ell}, \beta_{\ell}, \alpha_{\ell}\right) \in$ $T$ such that $\bigcup_{i=1}^{\ell} f r\left(\beta_{i}\right) \cup f r\left(\alpha_{i}\right)=\left\{x_{1}, \ldots, x_{k}\right\}$ holds is called a critical term.

If $\gamma$ is a critical term, let $\left(f_{1}, \beta_{1}, \alpha_{1}\right), \ldots,\left(f_{\ell}, \beta_{\ell}, \alpha_{\ell}\right)$ be the access terms used in its definition. For a state $S$ choose $b_{1}, \ldots, b_{k} \in B$ with $\operatorname{val}_{S, \zeta}\left(\beta_{i}\right)=\operatorname{val}_{S, \zeta}\left(\alpha_{i}\right)$ with $\zeta=\left\{x_{1} \mapsto b_{1}, \ldots, x_{k} \mapsto b_{k}\right\}$ for $i=1, \ldots, \ell$, and let $a=\operatorname{val}_{S,\left\{x_{1} \mapsto b_{1}, \ldots, x_{k} \mapsto b_{k}\right\}}(\gamma)$.

Definition 4. For a state $S$ of a database transformation $t$ let $C_{S}=\left\{v a l_{S}(\alpha) \mid\right.$ $\alpha \in T\} \cup\{$ true, false, $\perp\}$ and $B_{S}=\left\{a_{i} \mid f\left(a_{1}, \ldots, a_{n}\right) \in \operatorname{val}_{S}(\beta, \alpha)\right.$ for some access term $(f, \beta, \alpha) \in T\}$. Then $\bar{C}_{S}$ is the background closure of $C_{S} \cup B_{S}$ containing all complex values that can be constructed out of $C_{S} \cup B_{S}$ using the constructors and function symbols (interpreted in $S$ ) in the background. The elements of $\bar{C}_{S}$ are called the critical elements of $S$.

The following lemma and its proof are analogous to the result in [6, Lemma 6.2].

Lemma 1. For all updates $\left(f\left(a_{1}, \ldots, a_{n}\right), a_{0}\right) \in \Delta(t, S)$ the values $a_{0}, \ldots, a_{n}$ are critical elements of $S$.

In [6] it is a straighforward consequence of Lemma 6.2 that individual updates can be represented by assignments rules, and consistent update sets by parblocks of assignment rules. In our case showing that $\Delta(t, S)$ can be represented by a parDB-ASM rule requires a bit more work. We address this in the next lemma.

Lemma 2. Let $t$ be a database transformation. For every state $S \in \mathcal{S}_{t}$ there exists a rule $r_{S}$ such that $\Delta(t, S)=\Delta\left(r_{S}, S\right)$, and $r_{S}$ only uses critical terms.

Proof (sketch). Consider any update $u=\left(f\left(a_{1}, \ldots, a_{n}\right), a_{0}\right) \in \Delta(t, S)$. According to Lemma 1 the values $a_{0}, \ldots, a_{n}$ are critical and hence representable by terms involving variables from access terms in $T$, i.e. $a_{i}=\operatorname{val}_{S, \zeta}\left(t_{i}\right)$ with either $f r\left(t_{i}\right) \subseteq\left\{x_{1}, \ldots, x_{k}\right\}, \zeta=\left\{x_{1} \mapsto b_{1}, \ldots, x_{k} \mapsto b_{k}\right\}$ and

$$
\left(b_{1}, \ldots, b_{k}\right) \in \mathcal{B}_{u}=\left\{\left(b_{1}, \ldots, b_{k}\right) \in B^{k} \mid \bigwedge_{1 \leq i \leq \ell} \operatorname{val}_{S, \zeta}\left(\beta_{i}\right)=\operatorname{val}_{S, \zeta}\left(\alpha_{i}\right)\right\}
$$

with access terms $\left(f, \beta_{i}, \alpha_{i}\right) \in T(i=1, \ldots, \ell)$ and $f r\left(\beta_{i}\right) \subseteq\left\{x_{1}, \ldots, x_{k}\right\}$, or $t_{i}$ is a ground critical term.

As the case that all terms $t_{0}, \ldots, t_{n}$ are ground terms is straightforward, let us concentrate on the case that at least one of the terms $t_{0}, \ldots, t_{n}$ is not a ground 
term. We first assume that none of terms $t_{0}, \ldots, t_{n}$ contain location operators. The access terms $\left(\beta_{i}, \alpha_{i}\right)$ define a finite set of locations

$$
\begin{array}{r}
L=\left\{f\left(a_{1}, \ldots, a_{n}\right) \mid a_{i}=v_{a l} l_{S, \zeta}\left(t_{i}\right) \text { for } i=1, \ldots, n,\right. \text { and } \\
\left.\zeta=\left\{x_{1} \mapsto b_{1}, \ldots, x_{k} \mapsto b_{k}\right\} \text { for }\left(b_{1}, \ldots, b_{k}\right) \in \mathcal{B}_{u}\right\} .
\end{array}
$$

We may assume that the set $\mathcal{B}_{u}$ is minimal in the sense that we may not find additional access terms that would define a subset $\mathcal{B}_{u}^{\prime} \subsetneq \mathcal{B}_{u}$ still containing the value tuple $\left(b_{1}, \ldots, b_{k}\right)$ that is needed to define the update $u$. If $\ell$ is updated in $S$, then $\left(\ell, a_{0}\right)$ is represented by the assignment rule $f\left(t_{1}, \ldots, t_{n}\right):=t_{0}$ with $x_{1}, \ldots, x_{k}$ interpreted by $\left(b_{1}, \ldots, b_{k}\right) \in \mathcal{B}_{u}$.

Next we revise the previous assumption that none of terms $t_{0}, \ldots, t_{n}$ contain location operators to a general case, i.e. location operators may appear in the terms $t_{0}, \ldots, t_{n}$ of an assignment rule $f\left(t_{1}, \ldots, t_{n}\right):=t_{0}$. Let $f_{\ell}$ be a unary function symbol such that $x_{t_{i}}=f_{\ell}(i)$, then, without loss of generality, we can replace the terms $t_{1}, \ldots, t_{n}$ of an assignment rule $f\left(t_{1}, \ldots, t_{n}\right):=t_{0}$ with the variables $x_{t_{1}}, \ldots, x_{t_{n}}$, such that

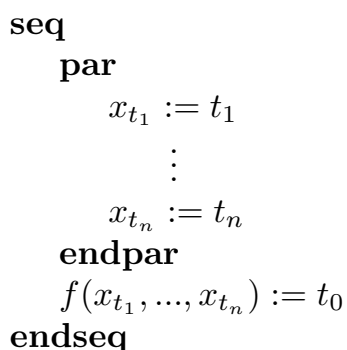

It means that we can simplify the construction of rules for updates which may correspond to terms with location operators by only considering the case that location operators appear at the right hand side of an assignment rule. If a term $t_{i}(i \in[1, n])$ at the left hand side contains a location operator, by the above translation, we may treat it as being a term at the right hand side of another assignment rule again.

Suppose that the outermost function symbol of term $t_{0}$ is a location operator $\rho$, e.g. $t_{0}=\rho(m)$ where $m=\left\langle t_{0}^{\prime}\right|$ for all values $\bar{a}=\left(a_{1}, \ldots, a_{p}\right)$ in $\bar{y}=\left(y_{1}, \ldots, y_{p}\right)$ such that $\operatorname{val}_{S, \zeta\left[x_{1} \mapsto b_{1}, \ldots, x_{k} \mapsto b_{k}\right]}(\varphi(\bar{x}, \bar{y}))=$ true $\rangle$, and $\bar{x}$ denotes a tuple of variables among $x_{1}, \ldots, x_{k}$. Then for each assignment rule $f\left(x_{t_{1}}, \ldots, x_{t_{n}}\right):=t_{0}$ in which $t_{0}$ contains a location operator as described before, we can construct the following rule to remove the location operator $\rho$ by a let rule and a forall rule:

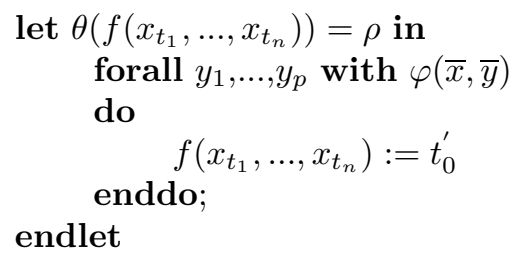


This construction can be conducted iteratively. If the outermost function symbol of the above term $t_{0}^{\prime}$ is a location operator, then we need to construct a rule in a similar way to replace the assignment rule $f\left(x_{t_{1}}, \ldots, x_{t_{n}}\right):=t_{0}^{\prime}$. This procedure continues until the right hand side of an assignment rule is a term without any location operator.

With Lemma 2 we have done the hard part of the proof. The following lemmata are almost identical to those in the proof of the DB-ASM thesis [7].

Lemma 3. Let $S, S^{\prime} \in \mathcal{S}_{t}$ be states that coincide on the set $C T$ of critical terms. Then $\Delta\left(r_{S}, S^{\prime}\right)=\Delta\left(t, S^{\prime}\right)$ holds.

Proof. As $S$ and $S^{\prime}$ coincide on $C T$, they also coincide on $T$, which gives $\Delta(t, S)=\Delta\left(t, S^{\prime}\right)$ by the bounded exploration postulate. Furthermore, we have $\Delta\left(r_{S}, S\right)=\Delta(t, S)$ by Lemma 2 . As $r_{S}$ uses only critical terms, the updates produced in state $S$ must be the same as those produced in state $S^{\prime}$, i.e. $\Delta\left(r_{S}, S\right)=$ $\Delta\left(r_{S}, S^{\prime}\right)$, which proves the lemma.

Lemma 4. Let $S, S_{1}, S_{2}$ be states with $S_{1}$ isomorphic to $S_{2}$ and $\Delta\left(r_{S}, S_{2}\right)=$ $\Delta\left(t, S_{2}\right)$. Then also $\Delta\left(r_{S}, S_{1}\right)=\Delta\left(t, S_{1}\right)$ holds.

Proof. Let $\sigma$ be an isomorphism from $S_{1}$ to $S_{2}$. Then $\Delta\left(r_{S}, S_{2}\right)=\sigma\left(\Delta\left(r_{S}, S_{1}\right)\right)$ holds, and the same applies to $\Delta\left(t, S_{2}\right)=\sigma\left(\Delta\left(t, S_{1}\right)\right)$. As we presume $\Delta\left(r_{S}, S_{2}\right)=$ $\Delta\left(t, S_{2}\right)$, we obtain $\sigma\left(\Delta\left(r_{S}, S_{1}\right)\right)=\sigma\left(\Delta\left(t, S_{1}\right)\right)$ and hence $\Delta\left(r_{S}, S_{1}\right)=\Delta\left(t, S_{1}\right)$, as $\sigma$ is an isomorphism.

Next, in the spirit of [6] we want to extend the equality of sets of update sets for $t$ and $r_{S}$ to a larger class of states by exploiting the finiteness of the bounded exploration witness $T$. For this we define the notion of $T$-equivalence similar to the corresponding notion for the sequential ASM thesis, with the difference that in our case we cannot take $T$, but must base our definition and the following lemma on $C T$.

Definition 5. States $S, S^{\prime} \in \mathcal{S}_{t}$ are called $T$-similar iff $E_{S}=E_{S^{\prime}}$ holds, where $E_{S}$ is an equivalence relation on $C T$ defined by

$$
E_{S}\left(\gamma_{1}, \gamma_{2}\right) \Leftrightarrow \operatorname{val}_{S}\left(\gamma_{1}\right)=\operatorname{val}_{S}\left(\gamma_{2}\right)
$$

Lemma 5. We have $\Delta\left(r_{S}, S^{\prime}\right)=\Delta\left(t, S^{\prime}\right)$ for every state $S^{\prime}$ that is $T$-similar to $S$.

Proof. Replace every element in $S^{\prime}$ that also belongs to $S$ by a fresh element. This defines a structure $S_{1}$ isomorphic to $S^{\prime}$ and disjoint from $S$. By the abstract state postulate $S_{1}$ is a state of $t$. Furthermore, by construction $S_{1}$ is also $T$-similar to $S^{\prime}$ and hence also to $S$.

Now define a structure $S_{2}$ isomorphic to $S_{1}$ such that $\operatorname{val}_{S_{2}}(\gamma)=\operatorname{val}_{S}(\gamma)$ holds for all critical terms $\gamma \in C T$. This is possible, as $S$ and $S_{1}$ are $T$-similar, i.e. we have $\operatorname{val}_{S}\left(\gamma_{1}\right)=\operatorname{val}_{S}\left(\gamma_{2}\right)$ iff $\operatorname{val}_{S_{1}}\left(\gamma_{1}\right)=\operatorname{val}_{S_{1}}\left(\gamma_{2}\right)$ for all critical terms $\gamma_{1}, \gamma_{2}$. By the abstract state postulate $S_{2}$ is also a state of $t$.

Using Lemma 3 we conclude $\Delta\left(r_{S}, S_{2}\right)=\Delta\left(t, S_{2}\right)$, and by Lemma 4 we obtain $\Delta\left(r_{S}, S^{\prime}\right)=\Delta\left(t, S^{\prime}\right)$ as claimed. 
We are now able to prove our main result, first generalising Lemma 2 to multiplesteps updates in the next lemma, from which the proof of the main characterisation theorem is straightforward.

Lemma 6 (sketch). Let $t$ be a synchronous parallel database transformation with signature $\Sigma$. Then there exists a parDB-ASM rule $r$ over $\Sigma$, with same background as $t$ such that $\Delta(r, S)=\Delta(t, S)$ holds for all states $S \in \mathcal{S}_{t}$.

Proof. In order to decide whether equivalence relations $E_{S}$ and $E_{S^{\prime}}$ coincide for states $S, S^{\prime} \in \mathcal{S}_{t}$ it is sufficient to consider the subset $C T^{\prime} \subseteq C T$ defined by the bounded exploration witness $T$ as in Definition 3 . Hence, as $T$ is finite, $C T^{\prime}$ is also finite, and consequently there can only be finitely many such equivalence relations. Let these be $E_{S_{1}}, \ldots, E_{S_{n}}$ for states $S_{1}, \ldots, S_{n} \in \mathcal{S}_{t}$.

For $i=1, \ldots, n$ construct Boolean terms $\varphi_{i}$ such that $\operatorname{val}_{S}\left(\varphi_{i}\right)=$ true holds iff $S$ is $T$-similar to $S_{i}$. For this let $C T^{\prime}=\left\{\gamma_{1}, \ldots, \gamma_{m}\right\}$, and define terms

$$
\bar{\gamma}_{j}= \begin{cases}\gamma_{j} & \text { if } \gamma_{j} \text { is closed } \\ \left\langle\left(x_{1}, \ldots, x_{k}\right) \mid \bigwedge_{1 \leq i \leq \ell} \beta_{i}=\alpha_{i}\right\rangle & \text { if } \gamma_{j}=\left(x_{1}, \ldots, x_{k}\right) \text { with variables taken } \\ & \text { from }\left(\beta_{1}, \alpha_{1}\right), \ldots,\left(\beta_{\ell}, \alpha_{\ell}\right)\end{cases}
$$

exploiting the fact that the background structures provide constructors for multisets and pairs (and thus also tuples). Then

$$
\varphi_{i}=\bigwedge_{\substack{1 \leq j_{1}, j_{2} \leq m \\ E_{S_{i}}\left(\gamma_{j_{1}}, \gamma_{j_{2}}\right)}} \bar{\gamma}_{j_{1}}=\bar{\gamma}_{j_{2}} \wedge \bigwedge_{\substack{1 \leq j_{1}, j_{2} \leq m \\ \neg E_{S_{i}}\left(\gamma_{j_{1}}, \gamma_{j_{2}}\right)}} \bar{\gamma}_{j_{1}} \neq \bar{\gamma}_{j_{2}}
$$

asserts that $E_{S}=E_{S_{i}}$ holds. Now define the rule $r$ by

$$
\begin{gathered}
\text { par if } \varphi_{1} \text { then } r_{S_{1}} \text { endif } \\
\text { if } \varphi_{2} \text { then } r_{S_{2}} \text { endif } \\
\vdots \\
\text { if } \varphi_{n} \text { then } r_{S_{n}} \text { endif endpar }
\end{gathered}
$$

If $S \in \mathcal{S}_{t}$ is any state of $t$, then $S$ is $T$-equivalent to exactly one $S_{i}(1 \leq i \leq n)$, which implies $\operatorname{val}_{S}\left(\varphi_{j}\right)=$ true iff $j=i$, and hence $\Delta(r, S)=\Delta\left(r_{S_{i}}, S\right)=\Delta(t, S)$ by Lemma 5 .

This completes the proof of Theorem 4 .

\section{Conclusion}

In this paper we generalise the DB-ASM thesis [7], i.e. the exact characterisation of database transformations by a variant of Abstract State Machines, to synchronous parallel database transformations. The key ideas in our previous definition of database transformations were 
- to capture the intrinsic finiteness of databases by adopting meta-finite structures 4] for the states,

- to capture the constructs for the data model by means of background structures,

- to capture aggregation in queries by particular multiset functions, and

- to permit special non-ground terms in bounded exploration witnesses to capture unbounded parallelism on the database part of a state.

The generalisation in this paper simply exploits the observation that the restriction of the extended non-ground terms in bounded exploration witnesses to the database part of the state signature is not necessary, while the other postulates remain unchanged.

As a consequence of the research presented in this paper we may first ask what what happens if we drop the request that states are meta-finite structures, and use simply any Tarski structures. This would mean to look only at the algorithmic part of the state. We would obtain a different thesis for synchronous parallel algorithms. The question to be investigated is how this thesis would differ from Blass' and Gurevich's parallel ASM thesis in [2], which is still debated in the ASM community. We conjecture that our result only provides a simplification of the postulates. Whether it is more intuitive has to be explored.

Naturally, synchronous parallel database transformations can be tailored to particular data models, as this only requires to adapt the background structures. For the case of XML the corresponding DB-ASMs have been investigated in detail in 8]. Furthermore, the complete logic for DB-ASMs (see 10] for a first version) should carry over to parallel DB-ASMs.

However, the DB-ASM thesis in [7] also supports bounded non-determinism, which for the sake of keeping the presentation simple was not handled in this paper. It should be possible to preserve the bounded non-determinism also for synchronous parallel database transformations, but the corresponding (more difficult) proofs have to be checked carefully.

With respect to the complete logic for DB-ASMs, however, we doubt that a generalisation would be straightforward (if possible at all), as our theory relies on the boundedness arising from the finiteness of the database part. Nonetheless it will be worth some research effort.

Finally, it appears to be much more challenging to approach also asynchronous parallel database transformations. We believe that in this case (different from some attempts by Yuri Gurevich) we cannot assume to have global states, so non-determinism becomes essential. We plan to investigate into this research direction.

\section{References}

1. Abiteboul, S., Hull, R., Vianu, V.: Foundations of Databases. Addison-Wesley Longman Publishing Co., Inc., Boston (1995)

2. Blass, A., Gurevich, Y.: Abstract state machines capture parallel algorithms. ACM Transactions on Computational Logic 4(4), 578-651 (2003) 
3. Börger, E., Stärk, R.: Abstract State Machines. Springer, Heidelberg (2003)

4. Grädel, E., Gurevich, Y.: Metafinite model theory. Information and Computation 140(1) (1998)

5. Gurevich, Y., Tillmann, N.: Partial updates. Theoretical Computer Science 336(23), 311-342 (2005)

6. Gurevich, Y.: Sequential abstract state machines capture sequential algorithms. ACM Transactions on Computational Logic 1(1), 77-111 (2000)

7. Schewe, K.-D., Wang, Q.: A customised ASM thesis for database transformations. Acta Cybernetica 19(4), 765-805 (2010)

8. Schewe, K.-D., Wang, Q.: XML database transformations. Journal of Universal Computer Science 16(20), 3043-3072 (2010)

9. Schewe, K.-D., Wang, Q.: Partial updates in complex-value databases. In: Heimbürger, A., et al. (eds.) Information and Knowledge Bases XXII. Frontiers in Artificial Intelligence and Applications, vol. 225, pp. 37-56. IOS Press (2011)

10. Wang, Q.: A Proof System with Bounded Non-determinism in Database Transformations. In: Schewe, K.D., Thalheim, B. (eds.) SDKB 2010. LNCS, vol. 6834, pp. 114-133. Springer, Heidelberg (2011) 